\title{
Rac1 mediates HMGB1-induced hyperpermeability in pulmonary microvascular endothelial cells via MAPK signal transduction
}

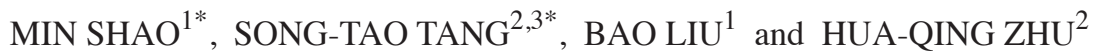 \\ ${ }^{1}$ Department of Critical Care Medicine, Affiliated Provincial Hospital of Anhui Medical University, Hefei, Anhui 230001; \\ ${ }^{2}$ Laboratory of Molecular Biology and Department of Biochemistry, Anhui Medical University, Hefei, Anhui 230032; \\ ${ }^{3}$ Department of Endocrinology, The First Affiliated Hospital of Anhui Medical University, \\ Hefei, Anhui 230022, P.R. China
}

Received December 22, 2014; Accepted September 16, 2015

DOI: $10.3892 / \mathrm{mmr} .2015 .4521$

\begin{abstract}
The pathology of acute respiratory distress syndrome (ARDS) is closely associated with the failure of alveolar-capillary barrier integrity and alveolar filling by high protein pulmonary edema, resulting from hyperpermeability. High mobility group box 1 (HMGB1) is a novel late mediator of sepsis, which is specifically involved in endotoxin-induced acute lung injury and sepsis-associated lethality. Although the role of HMGB1 in endothelial cell cytoskeletal rearrangement and vascular permeability have been investigated preliminarily, the molecular mechanisms remain to be fully elucidated. As the ras-related $\mathrm{C} 3$ botulinum toxin substrate 1 (Rac1) gene is important role in regulating microvascular barrier maintenance, the present study was designed to determine whether Rac1 is involved in HMGB1-induced hyperpermeability in pulmonary microvascular endothelial cells (PMVECs). The results of the present study demonstrated that HMGB1 induced dose and time-dependent decreases in transendothelial electrical resistance (TER). Notably, HMGB1 induced a dose-dependent increase in the activity and expression levels of Rac1. Using small interfering RNA and an agonist of Rac1, the present study demonstrated that Rac1 was a novel factor mediating the HMGB1-induced decrease in TER via extracellular signal-regulated kinase and p38 mitogen-activated
\end{abstract}

Correspondence to: Dr Bao Liu, Department of Critical Care Medicine, Affiliated Provincial Hospital of Anhui Medical University, 17 Lujiang Road, Hefei, Anhui 230001, P.R. China

E-mail: linux306@126.com

Dr Hua-Qing Zhu, Laboratory of Molecular Biology and Department of Biochemistry, Anhui Medical University, 81 Meishan Road, Hefei, Anhui 230032, P.R. China

E-mail: aydzhq@126.com

${ }^{*}$ Contributed equally

Key words: high mobility group box 1, ras-related C3 botulinum toxin substrate 1 , acute respiratory distress syndrome, hyperpermeability, mitogen-activated protein kinase protein kinase (MAPK) activation. These data suggested that Racl is involved in HMGB1-induced hyperpermeability in PMVECs via MAPK signal transduction.

\section{Introduction}

Acute respiratory distress syndrome (ARDS) is frequently associated with trauma, shock and sepsis, and is characterized by an intense inflammatory process involving the accumulation of activated neutrophils and the production of pro-inflammatory cytokines, which causes damage of alveolar-capillary integrity with subsequent high-permeability and non-hydrostatic pulmonary edema (1). The vital importance of the pulmonary microvascular barrier function is demonstrated by the balance between competing endothelial cell (EC) contractile forces and adhesive cell-cell and cell-matrix tethering forces, all of which are closely linked through the endothelial cytoskeleton (2). The tumor necrosis factor $\alpha$ (TNF- $\alpha$ ), interleukin (IL) and platelet-activating factor inflammatory factors, which induce pulmonary EC cytoskeletal rearrangement, increase paracellular gap formation and enhance microvascular permeability underlie the pathognomonic features of ARDS (1).

Ras homolog gene family, member A (RhoA), ras-related C3 botulinum toxin substrate 1 (Rac1) and cell division control protein 42 homolog (Cdc42), which belong to the Rho GTPase family, have been demonstrated to be important in the regulation of microvascular barrier maintenance by affecting the actin cytoskeleton (3). Evidence suggests that Rac and Rho have opposing roles in the regulation of barrier maintenance and stabilization. Waschke et al (4) demonstrated that acute inhibition of the small GTPase Rac1 caused a significant increase in the permeability of venular microvessels. Furthermore, various barrier-stabilizing mediators and the barrier-protective cyclic adenosine monophosphate-regulated, Rho, guanine nucleotide exchange factor (GEF) exchange protein directly activated by cAMP-1 have been shown to reduce microvascular permeability, at least in part, via the activation of $\operatorname{Racl}(5,6)$. It is also well-established that Racl is able to effectively stabilize the endothelial barrier via the strengthening of cortical actin, which can promote the stabilization of junctional proteins (7). However, unlike other factors in the microvascular endothelium, for example thrombin, vascular endothelial growth factor 
(VEGF) induces the activation of Racl and the rapid production of reactive oxygen species (ROS), causing endothelial barrier dysfunction (8). Furthermore, previous studies on the macrovascular endothelium have indicated that the activation of Rac1 may cause barrier destabilization, possibly through the generation of ROS (9-11). Therefore, whether Rac1 exerts protective or detrimental effects on barrier function depends on the types of cell and various inflammatory conditions.

High mobility group box 1 (HMGB1), originally identified as an important endogenous signaling molecule, is released by necrotic and inflammatory cells and has potent pro-inflammatory properties (12). HMGB1 induces the expression of adhesion molecules, including intercellular adhesion molecule 1 and vascular adhesion molecule 1 , and promotes the upregulation of pro-inflammatory cytokines, including TNF- $\alpha$, IL- 8 and plasminogen activator inhibitor 1, partly through the interaction of HMGB1 with its receptors (13-16). HMGB1 is different from early-acting mediators, including TNF- $\alpha$ and IL-1, and is recognized as a late-acting cytokine with a long duration of action, which is released with a lag phase of 16-24 h following endotoxin exposure (12). It is well-documented that HMGB1 contributes to the development of acute lung injury, and the underlying mechanisms may be associated with activation of the mitogen-activated protein kinase (MAPK) signaling pathway and nuclear factor- $\kappa \mathrm{B}$ via the interaction of HMGB1 with its receptors $(15,16)$.

It is noteworthy that, whether Rac1 is involved in HMGB1-induced hyperpermeability of the pulmonary microvascular endothelium and its associated molecular mechanisms remain to be fully elucidated. To investigate these gaps in current knowledge, the present study investigated pulmonary microvascular endothelial cells (PMVECs) with recombinant HMGB1, examining the role of HMCB1 and determining the mechanisms underlying the effects of Rac1 in HMGB1-mediated endothelial barrier function, which may provide novel therapeutic strategies in the treatment of ARDS via targeting Rac1.

\section{Materials and methods}

Reagents. Recombinant human HMGB1 was purchased from Sigma-Aldrich (St. Louis, MO, USA). The silencing RNA transfection reagent, siPORT ${ }^{\mathrm{TM}}$ Amine, was purchased from Ambion (Thermo Fisher Scientific, Waltham, MA, USA). The polyclonal rabbit anti-Rac1 (1:500; sc-217), monoclonal mouse anti-extracellular signal-regulated kinase (ERK) (1:500; sc-514302) and polyclonal rabbit anti-p38 (1:500; sc-535) antibodies were purchased from Santa Cruz Biotechnology, Inc. (Dallas, TX, USA). The monoclonal mouse anti-phosphorylated (p-)-ERK (1:500; 9101) and polyclonal rabbit anti-p-p38 $(1: 500 ; 9211)$ antibodies were purchased from Cell Signaling Technology, Inc. (Danvers, MA, USA). 8-CPT was purchased from Sigma-Aldrich. An enhanced chemiluminescence kit was obtained from Pierce Biotechnology, Inc. (Rockford, IL, USA).

Cell culture. The PMVECs were obtained from American Type Culture Collection (Manassas, VA, USA) and grown in the Endothelial Growth Medium-2 (Lonza Group Ltd., Basel, Switzerland) supplemented with growth factors including
VEGF, fibroblast growth factor, insulin growth factor-1 and epidermal growth factor (Gibco; Thermo Fisher Scientific), and $10 \%$ fetal bovine serum (Gibco; Thermo Fisher Scientific), according to the manufacturer's protocol. The cells were grown at $37^{\circ} \mathrm{C}$ in an atmosphere containing $5 \% \mathrm{CO}_{2}$, and those used for further experimentation were obtained from passage 6-9. For experimentation, the ECs were plated at an appropriate density, and used 3 days after plating, unless otherwise specified. Furthermore, the medium was replaced 1 day prior to all experiments. During experimentation, the PMVECs $\left(5 \times 10^{5}\right)$ were challenged with recombinant human HMGB1 at different doses $(5,10,15$ and $20 \mu \mathrm{g} / \mathrm{ml})$ for $2 \mathrm{~h}$. In addition, 8-CPT $(5 \mu \mathrm{M})$ was used to promote Rac1 in the PMVECs.

RNA interference. Dharmacon ON-TARGETplus small interfering RNA (siRNA) against Racl was obtained as pools of four siRNA duplexes from GE Healthcare Life Sciences, (Chalfont, UK). ON-TARGETplus siRNA (siControl) targeting a non-human protein, luciferase, was used as a negative control siRNA with minimal off-target silencing. The silencing protocol was optimized to allow transfection of the cells shortly following plating and on non-conventional substrates, including gold electrodes. At 3-5 h following cell plating, the siRNA, which was calculated at a final concentration of $100 \mathrm{nM}$ siRNA, was premixed with transfection reagent (4 $\mu \mathrm{l} / \mathrm{ml}$ siPORT ${ }^{\mathrm{TM}}$ Amine, Thermo Fisher Scientific) for $5 \mathrm{~min}$ and then diluted with basal media twice, based on using half the volume typical for a dish/well. After 16-24 h, an equal volume of serum media was added to the media containing siRNA. At 48 h post-transfection, the diluted siRNA media was replaced with serum media $(1 \mathrm{ml} /$ plate). The silenced cells were used 3-6 days post-transfection, and the media was replaced 1 day prior to all experiments.

Electrical resistance measurements. An electrical cell-substrate impedance sensing (ECIS) system (Applied Biophysics, Troy, NY, USA) was used to measure transendothelial electrical resistance (TER), using ECs grown on gold microelectrodes, as previously described by Wolfson et al (15). The ECs $\left(5 \times 10^{5}\right)$ were plated directly onto the gold microelectrodes of the ECIS arrays (8W10E), and cultured for a minimum of 2 days to establish confluency. Data pooling and analysis were performed using in-house-created Epool software (version 2.0), which has integrated graphing associated with Microsoft Excel (Microsoft, Redmond, WA, USA).

Racl activity assay. The activity levels of Rac1 were determined using a pull-down assay with an Rac Activation Assay kit (Cell Biolabs, Inc., San Diego, CA, USA), according to the manufacturer's protocol. Briefly, cells were lysed at $4^{\circ} \mathrm{C}$ in a pull-down lysis buffer. p21-activated kinase-conjugated protein beads were incubated with the cell lysates at $4^{\circ} \mathrm{C}$ for $1 \mathrm{~h}$. Eluted proteins were subjected to SDS-PAGE, followed by immunoblotting with the anti-Racl antibody.

Reverse transcription-quantitative polymerase chain reaction (RT-qPCR). SYBR ${ }^{\circledR}$ Green RT-qPCR (Takara Bio., Inc., Otsu, Japan) was used to detect mRNA expression levels. RNA was extracted from the cells using TRIzol reagent (Invitrogen; Thermo Fisher Scientific) and was reverse transcribed into 
cDNA $(1 \mu \mathrm{g})$ using a Prime Script RT Reagent kit (Takara Bio, Inc.) under the following conditions: $37^{\circ} \mathrm{C}$ for $15 \mathrm{~min}, 85^{\circ} \mathrm{C}$ for $5 \mathrm{sec}$ and $4^{\circ} \mathrm{C}$ for $1 \mathrm{~min}$. RT-qPCR was performed with ABI 7500 (Applied Biosystems; Thermo Fisher Scientific) using SYBR Premix Ex Taq (Takara Bio, Inc.) under the following thermocycling conditions: One cycle at $95^{\circ} \mathrm{C}$ for 30 min; 40 cycles at $95^{\circ} \mathrm{C}$ for $5 \mathrm{sec}$ and $60^{\circ} \mathrm{C}$ for $34 \mathrm{sec}$; one cycle at $95^{\circ} \mathrm{C}$ for $15 \mathrm{sec}, 60^{\circ} \mathrm{C}$ for $1 \mathrm{~min}$ and $95^{\circ} \mathrm{C}$ for $15 \mathrm{sec}$. The relative quantification values for these gene expression levels were calculated using the $\Delta \Delta \mathrm{Cq}$ (14) method and normalized using a house keeping gene. The following primer sequences were used: Rac1, forward 5'-GACCAGCCGACTAGCTTT TG-3' and reverse 5'-CGAAGGGATGCTCAAGAGAC-3'; GAPDH, forward 5'-AGGTCGGTGTGAACGGATTTG-3' and reverse 5'-GGGGTCGTTGAT GGCAACA-3' (Shanghai Shengong Biotechnology Co., Ltd., Shanghai, China). For cycling the ABI 7500 Real-Time PCR system (Thermo Fisher Scientific) was used.

Western blotting. The cells were washed once with cold endothelial basal medium (Lonza Group Ltd., Basel, Switzerland) and total protein was extracted using $0.3 \%$ SDS (Sigma-Aldrich) in $10 \mathrm{mM}$ Tris lysis buffer (300 $\mu$ l/D60; Sigma-Aldrich) containing protease and phosphatase cocktail inhibitors. The lysates were centrifuged at $14,000 \times \mathrm{g}$ for $30 \mathrm{~min}$ at $4^{\circ} \mathrm{C}$. The total protein concentration of each sample was measured using a MicroBCA Protein Assay Reagent kit (Pierce Biotechnology, Inc.). Lysates $(15 \mu \mathrm{l})$ from each line in the SDS sample buffer were then separated using 10\% SDS-PAGE (Bio-Rad Laboratories, Inc., Hercules, CA, USA) and electroblotted onto a polyvinylidene fluoride membrane (EMD Millipore, Billerica, MA, USA), which was then blocked with 5\% fat-free milk in phosphate-buffered saline with $0.1 \%$ Tween 20 (Sigma-Aldrich) for $1 \mathrm{~h}$ at room temperature. The membrane was then incubated with anti-Rac1, anti-phosphorylated (p-) ERK and anti-p-p38 overnight at $4^{\circ} \mathrm{C}$, followed by incubation with horseradish peroxidase-conjugated secondary antibodies (1:2,000; Beijing Zhongshan Jinqiao Biotechnology Co., Ltd., Beijing, China) for $2 \mathrm{~h}$ at room temperature. Specific bands of target proteins were stained with chemiluminescence reagent (Pierce Biotechnology, Inc.). Densitometric scanning of the exposed X-ray film (Kodak, Rochester, NY, USA) was used for semi-quantitative measurement of the protein bands. Target signals were normalized to those of GAPDH, and analyzed semi-quantitatively using a Quantity One analysis system (version 2.0; IBM Corporation, Armock, NY, USA).

Statistical analysis. All statistical analyses were performed using SPSS 13.0 for Windows (SPSS, Inc., Chicago, IL, USA). All data are presented as the mean \pm standard deviation. Comparisons between groups were performed using one-way analysis of variance and the Student-Newman-Keuls method. $\mathrm{P}<0.05$ was considered to indicate a statistically significant difference.

\section{Results}

HMGB1 induces dose- and time-dependent decreases in TER The present study measured the continuous TER of cultured PMVECs grown on gold microelectrodes. Challenge with
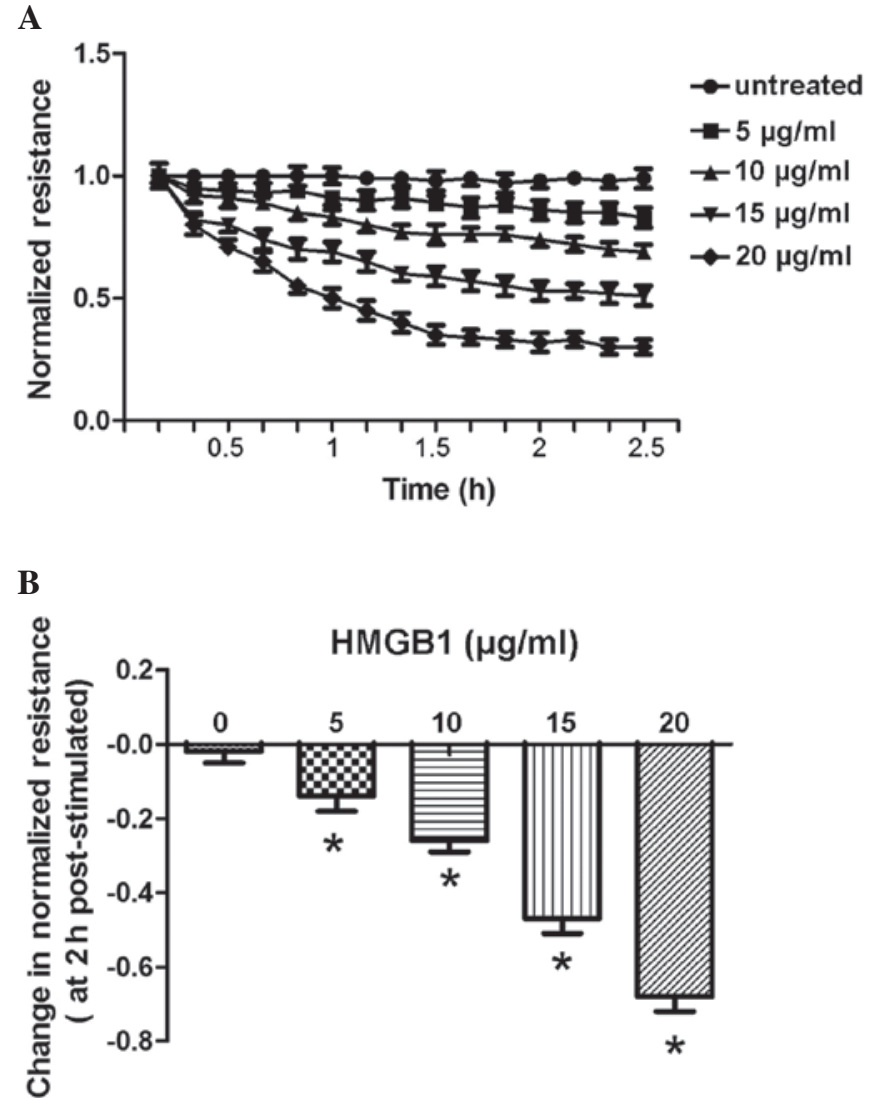

Figure 1. HMGB1 induces dose- and time-dependent decreases in TER (A) Cultured endothelial cells grown to confluency on gold electrodes were treated with recombinant HMGB1 $(5,10,15$ or $20 \mu \mathrm{g} / \mathrm{ml})$, and changes in TER were measured over time, between 0 and $2.5 \mathrm{~h}$. Decreased TER represents endothelial barrier dysfunction or increased vascular permeability. (B) Dose-dependent changes in resistance $2 \mathrm{~h}$ post-HMGB1 stimulation. Data are presented as the mean \pm standard deviation. ${ }^{*} \mathrm{P}<0.05$, vs. untreated control. TER, transendothelial electrical resistance; HMGB1, high mobility group box 1 .

recombinant HMGB1 produced dose-dependent $(5-20 \mu \mathrm{g} / \mathrm{ml})$ and time-dependent $(0-2.5 \mathrm{~h})$ decreases in TER measurements, indicating increased EC barrier dysfunction (Fig. 1A), compared with the control buffer, containing $20 \mathrm{mM}$ HEPES (pH 7.8), $150 \mathrm{mM} \mathrm{NaCl,} 0.2$ mM EDTA, 0.1\% Triton X-100, $2 \mathrm{mg} / \mathrm{ml}$ leupeptin and $0.1 \mathrm{mM}$ 4-(2-aminoethyl)-benzenesulfonyl fluoride. The results also demonstrated that the change in normalized resistance $2 \mathrm{~h}$ following stimulation was dose-dependent (5-20 $\mu \mathrm{g} / \mathrm{ml}$; Fig. 1B).

HMGB1 induces a dose-dependent increase in the expression and activity of Racl. To the best of our knowledge, there have been no reports regarding the association between HMGB1 and Racl, therefore, the present study aimed to determine whether HMGB1 regulated the expression and activity of Rac1. As shown in Fig. 2, the activity, mRNA expression and protein expression levels of Rac1 were significantly increased in a dose-dependent $(5-20 \mu \mathrm{g} / \mathrm{ml})$ manner following challenge of the PMVECs with HMGB1.

Racl is an important mediator of the HMGBl-induced TER decrease in PMVECs. The siRNA targeting Racl and the Rac1 agonist (8-CPT) were used to inhibit and induce the 
A

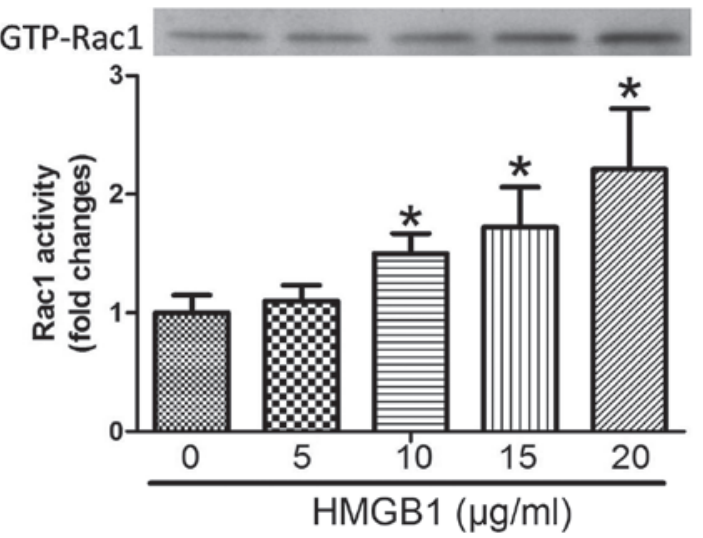

B

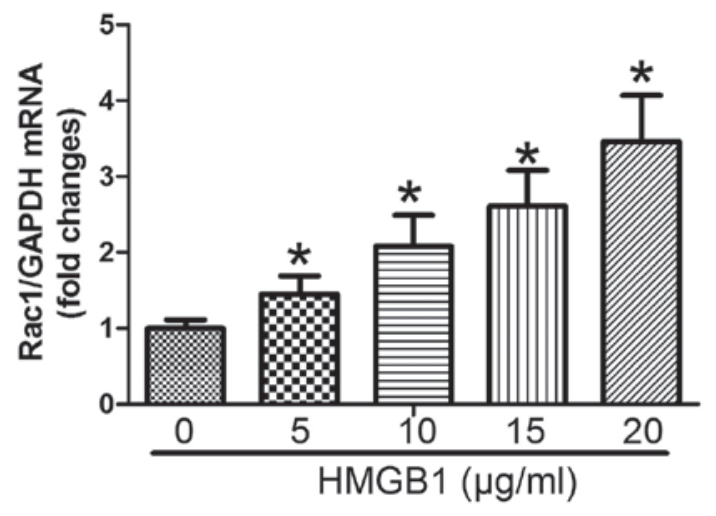

C
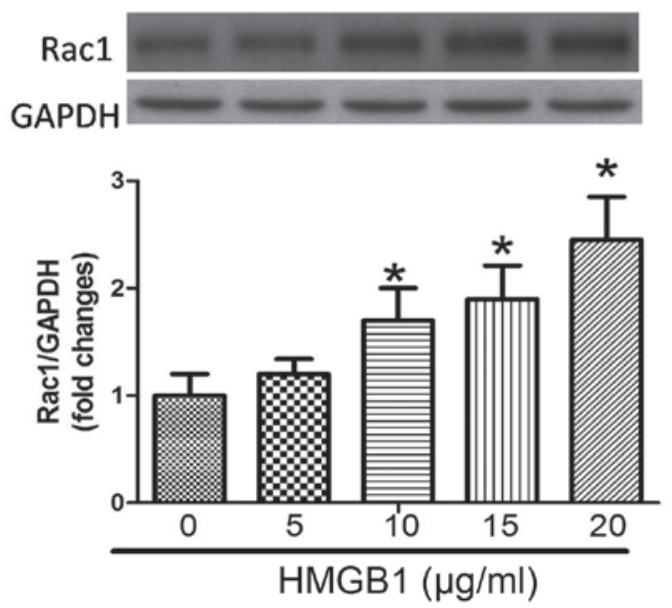

Figure 2. HMGB1 induces a dose-dependent increase in the expression and activity of Rac1. (A) Activity levels of Rac1 increased in a dose-dependent manner when the cultured endothelial cells were treated with recombinant HMGB1 $(5,10,15$ or $20 \mu \mathrm{g} / \mathrm{ml})$. (B) mRNA expression levels of Rac1 increased in a dose-dependent manner when the cultured endothelial cells were treated with recombinant HMGB1. (C) Protein expression levels of Racl increased in a dose-dependent manner when the cultured endothelial cells were treated with recombinant HMGB1. Data are presented as the mean \pm standard deviation, ${ }^{*} \mathrm{P}<0.05$, vs. untreated control. HMGB1, high mobility group box 1; Rac1, ras-related C3 botulinum toxin substrate 1 .

expression of Rac1 in PMVECs, respectively (Fig. 3A and B). The silenced and stimulated cells were subsequently plated on gold microelectrodes, allowing assay of TER following HMGB1 challenge $(20 \mu \mathrm{g} / \mathrm{ml})$. Downregulation of the expression of Rac1 significantly attenuated the HMGB1-induced decline in TER, whereas the overexpression of Rac1 significantly enhanced the HMGB1-induced decline in TER (Fig. 3C and D).
A
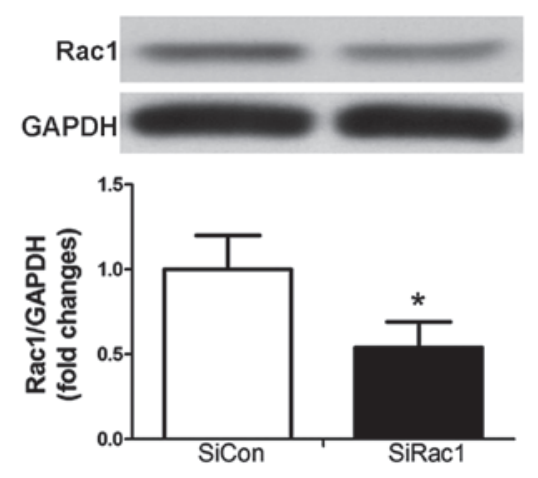

B
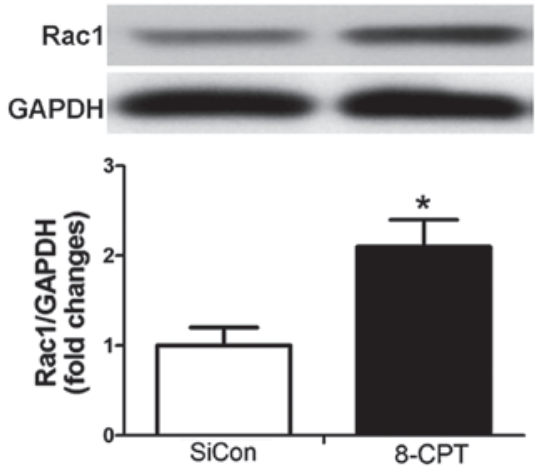

C

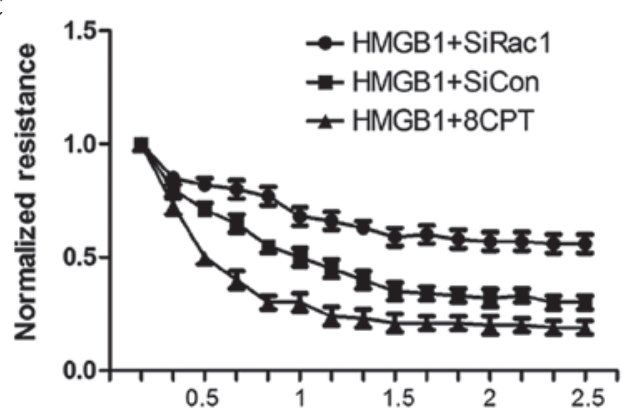

D

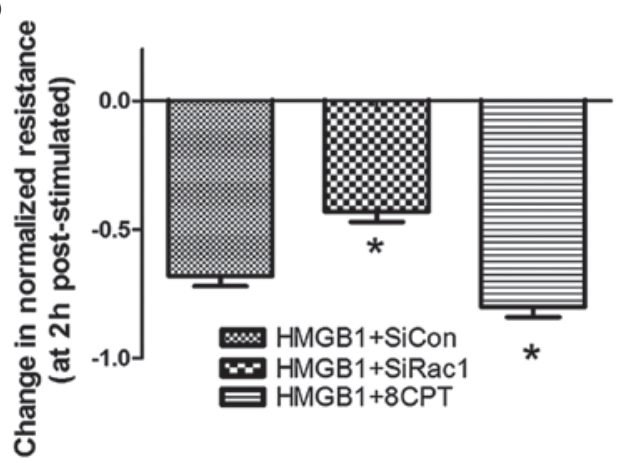

Figure 3. Rac1 mediates HMGB1-induced TER decrease in PMVECs. (A) Western blotting showed that siRacl inhibited the expression of Rac1, compared with the siCon-treated endothelial cells. (B) Western blotting revealed 8-CPT upregulated the expression of Rac1, compared with the siCon-treated cells. (C) Cultured endothelial cells grown to confluency on gold electrodes were treated with recombinant HMGB1 $(20 \mu \mathrm{g} / \mathrm{ml})$ and with either siRac1, siCon or 8-CPT. Changes in transendothelial electrical resistance were measured over time. (D) Changes in resistance $2 \mathrm{~h}$ post-stimulation. Data are presented with the mean \pm standard deviation. ${ }^{*} \mathrm{P}<0.05$, vs. siCon group. Con, control; si, small interfering; HMGB1, high mobility group box 1; Rac1, ras-related C3 botulinum toxin substrate 1 . 
A
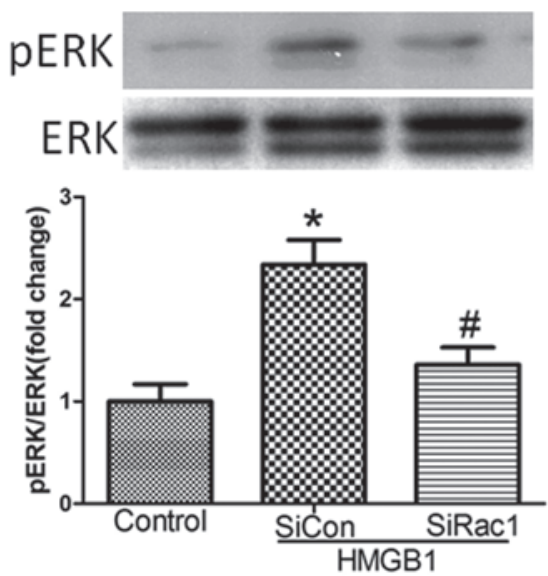

C
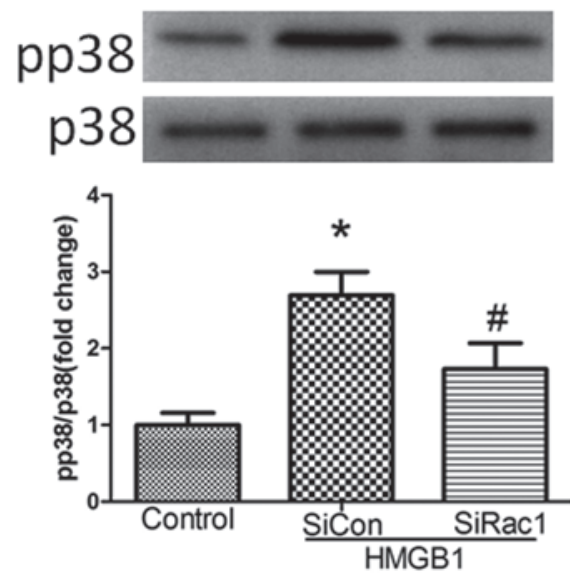

B
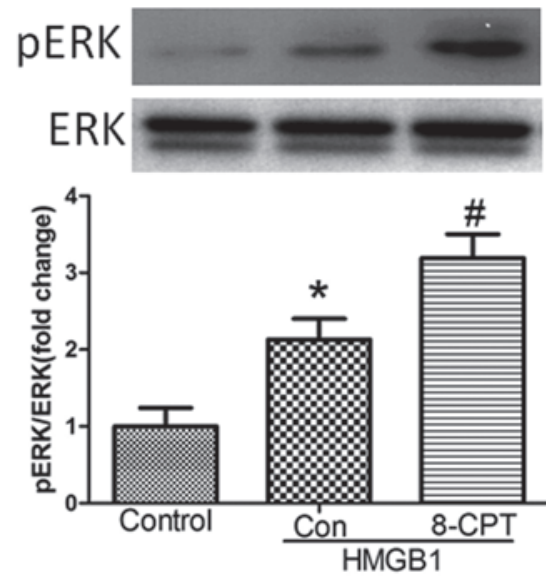

D
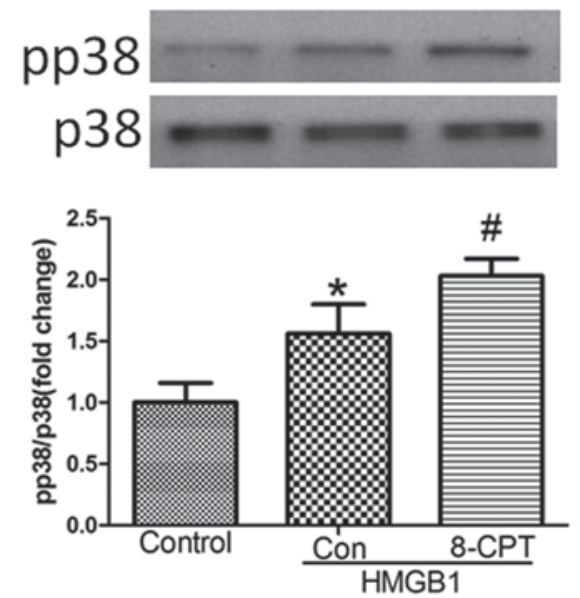

Figure 4. Rac1 mediates MAPK signaling following HMGB1 challenge. (A and B) Western blotting was performed to measure the effects of siRac1 and 8-CPT on the expression of pERK in cultured endothelial cells treated with recombinant HMGB1 ( $20 \mu \mathrm{g} / \mathrm{ml})$. (C and D) Western blotting was performed to measure the effects of siRacl and 8-CPT on the protein expression of p-p38 in cultured endothelial cells treated with recombinant HMGB1. Data are presented as the mean \pm standard deviation. ${ }^{*} \mathrm{P}<0.05$ and ${ }^{\#} \mathrm{P}<0.05$ vs. control. ERK, extracellular signal-regulated kinase; pERK, phosyphorylated ERK; pp38, phosphorylated p38; HMGB1, high mobility group box 1; si, small interfering.

Racl mediates MAPK signaling following HMGBl challenge. A previous study demonstrated that the MAPK signaling pathway mediated HMGB1-induced EC barrier disruption (15). The present investigation aimed to determine whether Rac1 mediated MAPK signaling following HMGB1 challenge $(20 \mu \mathrm{g} / \mathrm{ml})$. Treatment of the cells with siRacl or 8-CPT significantly inhibited and enhanced the expression of pERK, respectively, and increased the expression levels of p-p38 following HMGB1 challenge, compared with the control (Fig. 4).

\section{Discussion}

The molecular mechanisms underlying the contribution of HMGB1 to ARDS remain to be fully elucidated. The present study further demonstrated several molecular events, which are involved in HMGB1-induced hyperpermeability. The results of the present study indicated that HMGB1 induced dose- and time-dependent decreases in TER, and these results are concordant with those of a previous study (14). Notably, HMGB1 induced a dose-dependent increase in the activity and expression levels of Rac1. In addition, following the use of siRNA and an agonist of Rac1, the results of the present study demonstrated that Racl was a novel factor mediating the HMGB1-induced decrease in TER via ERK and p38 MAPK activation.

Widespread injury to the lung and systemic endothelium, resulting in high-permeability pulmonary edema, is a typical characteristic of ARDS, which was first recognized in ultrastructural investigations almost 30 years ago (1). Disruption of the semi-permeable barrier, which is formed by the pulmonary endothelium, results in a marked increase in the levels of fluid and protein leaving the vascular space through the alveolar epithelium into the interstitium and airspaces (1). The integrity of the pulmonary endothelium barrier is regulated by competing EC contractile forces and adhesive cell-cell tethering forces, which are closely associated with the endothelial actin cytoskeleton. Disruption of actin increases permeability and damages barrier integrity (2). Increasing evidence has suggested that HMGB1, a late-acting cytokine mediating endotoxin-associated lethality, causes reorganization of the actin cytoskeleton and disruption of an important junctional protein, vascular endothelial-cadherin, and induces paracellular gap formation in human pulmonary artery endothelial 
cells (15). In the present study, HMGB1 induced a marked decrease in TER, reflecting increased barrier dysfunction in PMVECs, and these results are similar to those of a previous study (15).

There is an increasing body of evidence suggesting that Rac1, Cdc42 and Rap1 contribute to the maintenance and stabilization of microvascular endothelial barrier functions, whereas RhoA primarily acts adversely to impair barrier integrity $(9,17)$. In previous years, there has been substantial attention on the role of Rac in the assembly of inter-endothelial junctions, and its increase in activity during junction formation (3). In the absence of vasoactive stimuli, Rac1 deficiency increases endothelial permeability and cannot form lamellipodial structures, focal adhesions or cell-cell contacts due to adherens and tight junction dysfunction, indicating that $\mathrm{Rac}$ is important for maintaining cell-cell junctions $(10,18)$. Furthermore, a previous study demonstrated that treatment with NSC-23766, which disturbs the Rac1-specific GEFs T-cell lymphoma invasion and metastasis 1 and Trio, also decreased TER and promoted intercellular gap formation (19). Our previous study also demonstrated that TNF- $\alpha$-induced PMVECs barrier breakdown was, at least in part, mediated by Rac1 inactivation (20). In addition, as the majority of findings on the microvascular endothelium are in accordance with existing reports that macrovascular endothelial cells are negatively affected by constitutively inactive Racl mutants $(9,18)$, it is well-established that Racl appears to improve endothelial barrier properties under resting conditions. However, several investigations have reported different results. In vitro, unlike thrombin, VEGF has been observed to induce rapid activation of Rac1 and increase the production of ROS, resulting in endothelial barrier dysfunction (8). The activation of Rac1 in the macrovascular endothelium may induce barrier destabilization, possibly via the generation of ROS (9-11). Therefore, the protective or deleterious role of Rac1 activation in microvascular endothelial barrier function is dependent on cell type and various stimuli. In the present study, the results demonstrated that the activity and expression levels of Rac1 in PMVECs were elevated when stimulated by HMGB1, and Rac1 acted as a promoter of HMGB1-induced barrier dysfunction and endothelial hyperpermeability. These findings indicate a preliminary role for Rac1 in HMGB1-induced barrier dysfunction in PMVECs, and requires further investigation in the future.

The signaling pathways involved in HMGB1-induced barrier dysfunction have been investigated in previous studies. Wolfson et al (15) demonstrated that the receptor for advanced glycation end products (RAGE) was the primary receptor signaling HMGB1-induced TER decrease and paracellular gap formation. Of note, ECs treated with AGE-bovine serum albumin show increased permeability and actin cytoskeleton rearrangement, and these effects were alleviated following treatment with anti-RAGE antibody, specific MAPK inhibitors or dominant negative forms of ERK and p38 MAPK, indicating that the MAPK signaling pathway may be involved in HMGB1/RAGE-induced barrier dysfunction (21). Wolfson et al (15) also reported that pre-treatment with SB203580 resulted in the attenuation of HMGB1-induced TER disruption, and p38 MAPK was the direct downstream target of HMGB1/RAGE. In the present study, the results further demonstrated that HMGB1 stimulated the expression of p-ERK and p-p38 in PMVECs. A previous study suggested that Rac induces MAPK signaling under several conditions. Lin et al (22) reported that treatment of human lung fibroblasts with CXCL12 caused the activation of Rac1, Rho and ERK, and the CXCL12-induced increase in ERK phosphorylation was inhibited by RacN17, a dominant negative mutant of Rac1. Using siRNA and an agonist of Rac1, the present study also demonstrated that Rac1 promoted ERK/p38 MAPK signaling in the PMVECs following challenge with HMGB1. Therefore, HMGB1 induced hyperpermeability in PMVECs via the Rac1/MAPK signaling pathway.

In conclusion, the results of the present study confirmed the effects of HMGB1 on endothelial barrier function via measurement of TER, a reflection of loss of barrier integrity. The underlying mechanisms may, at least in part, be through Rac1-mediated MAPK signal pathways being involved in HMGB1-induced hyperpermeability. Future investigations may further elucidate the precise mechanisms underlying HMGB1-induced ARDS, and thereby provide novel therapeutic approaches in the treatment of ARDS.

\section{Acknowledgements}

The present study was supported by the National Natural Science Foundation of China (grant nos. 81070232 and 81270372) and the Natural Science Foundation of Anhui Province (grant no. 1408085MH170).

\section{References}

1. Ware LB: Pathophysiology of acute lung injury and the acute respiratory distress syndrome. Semin Respir Crit Care Med 27: 337-349, 2006.

2. Dudek SM and Garcia JG: Cytoskeletal regulation of pulmonary vascular permeability. J Appl Physiol (1985) 91: 1487-1500, 2001.

3. Beckers CM, van Hinsbergh VW and van Nieuw Amerongen GP: Driving Rho GTPase activity in endothelial cells regulates barrier Integrity. Thromb Haemost 103: 40-55, 2010.

4. Waschke J, Baumgartner W, Adamson RH, Zeng M, Aktories K, Barth H, Wilde C, Curry FE and Drenckhahn D: Requirement of Rac activity for maintenance of capillary endothelial barrier properties. Am J Physiol Heart Circ Physiol 286: H394-H401, 2004.

5. Adamson RH, Ly JC, Sarai RK, Lenz JF, Altangerel A, Drenckhahn D and Curry FE: Epac/Rap1 pathway regulates microvascular hyperpermeability induced by PAF in rat mesentery. Am J Physiol Heart Circ Physiol 294: H1188-H1196, 2008.

6. Birukova AA, Fu P, Xing J and Birukov KG: Rap1 mediates protective effects of iloprost against ventilator-induced lung injury. J Appl Physiol (1985) 107: 1900-1910, 2009.

7. Spindler V, Schlegel N and Waschke J: Role of GTPases in control of microvascular permeability. Cardiovasc Res 87: 243-253, 2010

8. Wang Y, Zang QS, Liu Z, Wu Q, Maass D, Dulan G, Shaul PW, Melito L, Frantz DE, Kilgore JA, et al: Regulation of VEGF-induced endothelial cell migration by mitochondrial reactive oxygen species. Am J Physiol Cell Physiol 301: C695-C704, 2011.

9. Wojciak-Stothard B, Tsang LY and Haworth SG: Rac and Rho play opposing roles in the regulation of hypoxia/reoxygenation-induced permeability changes in pulmonary artery endothelial cells. Am J Physiol Lung Cell Mol Physiol 288: L749-L760, 2005.

10. van Wetering S, van Buul JD, Quik S, Mul FP, Anthony EC, ten Klooster JP, Collard JG and Hordijk PL: Reactive oxygen species mediate Rac-induced loss of cell-cell adhesion in primary human endothelial cells. J Cell Sci 115: 1837-1846, 2002. 
11. Chen W, Pendyala S, Natarajan V, Garcia JG and Jacobson JR Endothelial cell barrier protection by simvastatin: GTPase regulation and NADPH oxidase inhibition. Am J Physiol Lung Cell Mol Physiol 295: L575- L583, 2008.

12. Wang H, Bloom O, Zhang M, Vishnubhakat JM, Ombrellino M Che J, Frazier A, Yang H, Ivanova S, Borovikova L, et al: HMG-1 as a late mediator of endotoxin lethality in mice. Science 285 248-251, 1999.

13. Kim DC, Lee W and Bae JS: Vascular anti-inflammatory effects of curcumin on HMGB1-mediated responses in vitro. Inflamm Res 60: 1161-1168, 2011.

14. Gao M, Hu Z, Zheng Y, Zeng Y, Shen X, Zhong D and He F: Peroxisome proliferator-activated receptor $\gamma$ agonist troglitazone inhibits high mobility group box 1 expression in endothelial cells via suppressing transcriptional activity of nuclear factor $\kappa \mathrm{B}$ and activator protein 1. Shock 36: 228-234, 2011.

15. Wolfson RK, Chiang ET and Garcia JG: HMGB1 induces human lung endothelial cell cytoskeletal rearrangement and barrier disruption. Microvasc Res 81: 189-197, 2011.

16. Kim JY, Park JS, Strassheim D, Douglas I, Diaz del Valle F Asehnoune K, Mitra S, Kwak SH, Yamada S, Maruyama I, et al: HMGB1 contributes to the development of acute lung injury after hemorrhage. Am J Physiol Lung Cell Mol Physiol 288 L958-L965, 2005.
17. Wojciak-Stothard B and Ridley AJ: Rho GTPases and the regulation of endothelial permeability. Vascul Pharmacol 39: 187-199, 2002.

18. Wójciak-Stothard B, Potempa S, Eichholtz T and Ridley AJ: Rho and Rac but not Cdc42 regulate endothelial cell permeability. J Cell Sci 114: 1343-1355, 2001.

19. Baumer Y, Spindler V, Werthmann RC, Bünemann M and Waschke J: Role of Rac 1 and cAMP in endothelial barrier stabilization and thrombin-induced barrier breakdown. J Cell Physiol 220: 716-726, 2009.

20. Shao M, Yue Y, Sun GY, You QH, Wang N and Zhang D: Caveolin-1 regulates Rac1 activation and rat pulmonary microvascular endothelial hyperpermeability induced by TNF- $\alpha . P L o S$ One 8: e5521, 2013.

21. Guo XH, Huang QB, Chen B, Wang SY, Li Q, Zhu YJ, Hou FF, Fu N, Brunk UT and Zhao M: Advanced glycation end products induce actin rearrangement and subsequent hyperpermeability of endothelial cells. APMIS 114: 874-883, 2006.

22. Lin $\mathrm{CH}$, Shih $\mathrm{CH}$, Tseng CC, Yu CC, Tsai YJ, Bien MY and Chen BC: CXCL12 induces connective tissue growth factor expression in human lung fibroblasts through the Rac1/ERK, JNK, and AP-1 pathways. PLoS One 9: e104746, 2014. 